\title{
Spinal cord repair: future directions
}

\author{
J W Fawcett \\ Physiological Laboratory, Downing Street, Cambridge, CB2 3EG, England.
}

\begin{abstract}
The main cause of disability following spinal injury is failure of axons to regenerate and reconnect the spinal cord with the brain. If patients with cord lesions are ever to make a full recovery some means will have to be found to restore ascending sensory and descending motor connections. Until the last few years there has been a very limited understanding of the reasons why axons in the central nervous system (CNS) fail to regenerate, but as a result of recent work the picture is now much clearer.
\end{abstract}

Key words: spinal cord; astroglia; oligodendrocyte; schwann cell; neurotrophic factors; axon regeneration.

\section{Why do axons fail to regenerate in the CNS?}

The CNS environment is inhibitory to axon growth: this can be shown by transplanting CNS tissue into peripheral nerves where it forms a barrier to axon regeneration, or by observing sensory axons regenerating in the dorsal root towards the spinal cord, where they cease to grow exactly where they encounter CNS tissue. ${ }^{1-3}$ Peripheral nerve (PNS) tissue on the other hand strongly promotes axon regeneration, even from CNS neurons. Thus if a peripheral nerve graft is inserted into the CNS, CNS neurons which would never normally regenerate their axons do so for a considerable distance in the Schwann cell environment of the peripheral nerve. ${ }^{4}$ What is the cause of the inhibitory nature of the CNS environment? There appear to be 2 main factors. First, oligodendrocytes have on their surface molecules which are inhibitory to axonal growth cones. ${ }^{5}$ Second, astrocytic tissues, as found in glial scars, are extremely hard for axons to penetrate, for reasons which are not yet fully established. ${ }^{6}$ In addition to CNS tissue discouraging axon regeneration, neurons themselves change, so that axons regenerating from adult neurons grow much less vigorously than axons from embryonic neurons: ${ }^{7}$ the greater vigour of growth from embryonic neurons is one of the reasons why brain grafts are only successful if derived from embryonic donors. ${ }^{8}$

\section{What types of treatment are under development?}

The 3 causes of failure of regeneration in the $\mathrm{CNS}$ are therefore the inhibitory surface of oligodendrocytes, the resistance of astrocytes to penetration by axons, and the decrease in the vigour of axon growth with age. What treatments are available which might neutralise or ameliorate these effects? The inhibitory effect of oligodendrocytes on axons is due to 2 partially identified molecules of 35 and $250 \mathrm{kD}$, whose effects in vitro can be neutralised by monoclonal antibodies. When these antibodies are applied to the lesioned rat spinal cord in vivo, some axons can be seen to regenerate past the lesion for distances of over $1 \mathrm{~cm}$, much further than in control animals. Although the number of axons regenerating is too few to have much functional effect, the antibodies are clearly making the CNS environment less inhibitory to axon growth. ${ }^{5}$ The reasons for the blocking of axon growth by astrocytic scars are still not fully understood, and this makes it difficult to design treatments to reverse the effect. However, preliminary results from our laboratory suggest that some trophic agents active on 
astrocytes may serve to reduce their blocking effects. Equally, it is not known what changes in gene expression underlie the reduced vigour of axon growth with age, but again it has been shown that some trophic agents such as NGF, FGF and BDNF are able to boost axon regeneration in vitro. ${ }^{9,10}$

Each of the 3 inhibitory effects can therefore be ameliorated to some extent. The question is whether any of these treatments have yet reached the stage where they will be of benefit to patients with spinal injuries. It is clear that none of the currently available treatments by itself is sufficient to induce spinal cord repair. However a combination of treatments may be more effective. Schwab has applied a combination of treatments to the crushed optic nerve: antibodies to block oligodendrocyte inhibition and FGF to the retina to enhance neurite outgrowth, and has seen significant axonal regeneration (unpublished results). If factors active on astrocytes were added as well, even more regeneration would probably result. It is clear, therefore, that any treatment designed to enhance axon regeneration in the injured spinal cord based on current knowledge of the field, will have to involve several active agents applied to the site of injury and elsewhere, and any treatment regimen is therefore going to be complex. However, it is probable that multiple treatments of this type can be developed reasonably soon to the stage where they allow axon regeneration over moderate distances in the spinal cord of experimental animals, but it is unlikely that any procedure based on our current knowledge will allow complete repair.

\section{How might the treatments be used?}

How might such combination treatments be used? Patients with subtotal spinal cord transection can have surprisingly normal function, despite having a greatly reduced number of descending and ascending axons. A small number of the right kind of axons regenerating to the right place might therefore be of considerable functional benefit. This might be arranged by insertion of a Schwann cell or peripheral nerve graft, positioned so as to channel a small number of axons from a point proximal to the injury to a particular level of the cord distal to the injury. The limitations of such grafts at present are that axons that grow through the graft cannot penetrate far enough into spinal cord tissue distal to the graft to make useful connections, and that the long axons from motor cortex and other higher centres tend to regenerate poorly in them. ${ }^{11}$ These problems can probably be partially alleviated by a combination of treatments: neurotrophic factors to neuronal cell bodies and axons to enhance outgrowth, blockers of oligodendrocyte inhibition and astrocyte active factors to make it easier for regenerating axons leaving the graft to penetrate the spinal cord and make connections with appropriate spinal neurons. Trophic factors and blocking antibodies, being polypeptides, are unable to cross the blood brain barrier, and have a limited lifetime, which makes them very difficult to administer. Many pharmaceutical companies are presently working to develop trophic factor agonists which will be much easier to handle, and would make treatment much more practicable.

Multiple treatments of the type outlined above are only starting to be investigated in animals, and will need some work before they are optimised. Practical application in spinally injured humans will be extremely difficult, and success will probably come in small increments; a sudden therapeutic leap to complete spinal cord regeneration seems unlikely based on current scientific progress.

\section{References}

1 Aguayo AJ, Dickson R, Trecarten J, Attiwell M (1978) Ensheathment and myelination of regenerating PNS fibers by transplanted optic nerve glia. Neurosci Lett 9: 97-104.

2 Weinberg EL, Spencer PS (1979) Studies on the control of myelinogenesis. 3. Signalling of oligodendrocyte myelination by regenerating peripheral axons. Brain Res 162: 273-279.

3 Carlstedt T, Cullheim S, Risling M, \& Ulfhake B (1989) Nerve fibre regeneration across the PNS-CNS interface at the root-spinal cord junction. Brain Res Bull 22: 93-102. 
4 David, S, Aguayo AJ (1981) Axonal elongation into peripheral nervous system bridges after central nervous system injury in adult rats. Science 241: $931-933$.

5 Schwab ME (1990) Myelin-associated inhibitors of neurite growth and regeneration in the CNS. TINS 13 452-456.

6 Fawcett JW, Housden E, Smith-Thomas L, Meyer RL (1989) The growth of axons in three dimensional astrocyte cultures. Dev Biol 135: 449-458.

7 Fawcett JW (in press) Intrinsic neuronal determinants of axon growth and regeneration. TINS.

8 Bjorklund A, Stenevi U (1984) Intracerebral neural implants: neuronal replacement and reconstruction of damaged circuitries. Ann Rev Neurosci 7: 279-308.

9 Lindsay RM (1988) Nerve growth factors (NGF, BDNF) enhance axonal regeneration but are not required for survival of adult sensory neurons. $J$ Neurosci 8: 2394-2405.

10 Bähr M, Vanselow J, Thanos S (1989) Ability of adult rat ganglion cells to regrow axons in vitro can be influenced by fibroblast growth factor and gangliosides. Neurosci Lett 96: 197-201.

11 Kuhlengel KR, Bunge MB, Bunge RP, Burton H (1990) Implantation of cultured sensory neurons and Schwann cells into lesioned neonatal rat spinal cord. II. Implant characteristics and examination of corticospinal tract growth. J Comp Neurol 293: 74-91. 